\title{
Associated Factors of Cardiovascular Diseases in Pakistan: Assessment of Path Analyses Using Warp Partial Least Squares Estimation
}

\author{
Mirza Rizwan Sajid ${ }^{1}$, Noryanti Muhammad ${ }^{2 *}$, Roslinazairimah Zakaria ${ }^{3}$ \\ Ahmad Shahbaz ${ }^{4}$, Ahmad Nauman ${ }^{5}$ \\ * Corresponding Author
}

1. Centre for Mathematical Sciences, College of Computing and Applied Sciences, Universiti Malaysia Pahang, 26300 Gambang, Kuantan, Pahang Darul Makmur, Malaysia, mirzarizwansajid@ gmail.com

2. Centre for Mathematical Sciences, College of Computing and Applied Sciences, Universiti Malaysia Pahang, 26300 Gambang, Kuantan, Pahang Darul Makmur, Malaysia, noryanti@ump.edu.my

3. Centre for Mathematical Sciences, College of Computing and Applied Sciences, Universiti Malaysia Pahang, 26300 Gambang, Kuantan, Pahang Darul Makmur, Malaysia, roslinazairimah@ump.edu.my

4. Punjab Institute of Cardiology, Pakistan, drahmadshahbaz@gmail.com

5. Punjab Institute of Cardiology, Pakistan, ahmadnoeman@gmail.com

\begin{abstract}
Demographic and socio-economic (SE) factors are associated with modifiable, clinical factors and cardiovascular disease (CVDs). However, the inclusion of mediators in these relationships creates complex pathways which extend the roles of factors within the model. The traditional hierarchal logistic regression (HLR) is unable to estimate the transmittal effects of factors through different layers of factors of CVDs. The study aims to simultaneously estimate and validate the probable linear and non-linear relationships among factors of CVDs considering potential mediators. Four hundred sixty participants (312 males and 148 females) were selected through systematic sampling in this sex-matched case control (1:1 ratio) study conducted in the largest Cardiac Center of Pakistan. The information on demographic, SE, modifiable and clinical factors of CVDs was recorded. Warp partial least squares (PLS) based on warp 3 algorithm was used to estimate the simultaneous linear and non-linear path coefficients of the proposed model of study. The study found that demographic and SE factors played a significant role in shaping the modifiable factors which further transmit their impact to CVDs. However, this transmitted impact of modifiable factors on CVDs was mediated through metabolic syndrome abnormalities (MSA) except self-reported subjective stress (SSS). Sleep satisfaction and negative dietary habits were the mediators between the relationship of SSS and MSA. Physical activity is the strongest factor associated with CVDs status. The proposed path analyses, verifying the mediation role of MSA in the pathways of relationships which would help in identifying the risky group of population and guide in formulating the health promotion policies for the reduction of CVDs burden. Further, Warp 3 algorithm is the better option to estimate complex models containing linear and non-linear relationship in the same model.
\end{abstract}

Key Words: Cardiovascular Diseases; Risk Factors; Path Analyses; Partial Least Squares; Warp 3 Algorithm; Mediation.

\section{Introduction}

The role of different forms of risk factors in cardiovascular diseases (CVDs) has been widely investigated through association studies and predictive models. Modifiable risk factors like smoking (Chen et al., 2008), alcohol consumption (Xi et al., 2017), disturbed sleeping patterns (Cappuccio, Cooper, D'elia, Strazzullo, \& Miller, 2011), stressful life (Song et al., 2019), negative dietary habits (NDH) (Menotti, Alberti-Fidanza, Fidanza, Lanti, \& Fruttini, 2012) and physical inactivity (Thivel et al., 2018) are the empirically proven risk factors of CVDs. Further, some modifiable abnormalities which are high fasting glucose, lipid abnormality, hypertension and obesity are also strongly associated with CVDs outcome (Alberti et al., 2009). The clustering of these abnormalities (with some disagreement in the classification) is recognized as metabolic syndrome (MS). Additionally, background factors (demographic and 
socio-economic) are associated with these modifiable risk factors and CVDs as well (Kastorini et al., 2015; M. Sajid, Ansar, Hanif, Waheed, \& Tufail, 2017) that reflected the multifaceted nature of these background and modifiable risk factors in the development of CVDs. It reveals that this is not a cause and effect phenomenon but containing multiple pathways for effect transference and needs complex modeling. Therefore, there is a need of exploratory approach that can model the causes of the causes rather than focusing on simple cause and effect phenomenon (Braveman \& Gottlieb, 2014) that could help in designing targeted and effective interventions for CVDs reduction.

There is a scarcity of literature about complex modeling in the Pakistani context which is facing $29 \%$ deaths due to CVDs (Organization, 2018). The exploratory approach mentioned above is not only elucidating the drift of relationships but also escalating the complexity in the aforementioned phenomenon through mediating roles of factors especially modifiable risk factors and MS. Surprisingly, the role of MS as a mediator has not been examined in the overall literature except a review paper which suggested mediating role of MS in the relationship of demographic factors and CVDs (Muhammad, Sajid, Zakaria, \& Shahbaz, 2019). Further, there is also little published research on the mediating role of modifiable factors (stress, diet, physical activity and smoking) in the CVDs (Kastorini et al., 2015). Most of the work predominantly concerned with the direct relationship between modifiable risk factors and MS (Hung et al., 2013; Misra \& Khurana, 2009) or MS and CVDs (Grundy, 2016) and failed to consider the potential indirect impact of modifiable factors on CVDs through MS. Further, age and gender can cofound the relationships in the model (Kastorini et al., 2015). This interplay of numerous types of factors has created multiple novel pathways of the relationship between them that leads to parameters estimation issues through probability models that could not manage the residual confounding and mediating effects of risk factors (Silva, Vasconcelos, Bettiol, \& Barbieri, 2010). Based on above-mentioned gaps in the literature and possible associated factors of CVDs the conceptual model is given in Figure 1.

In epidemiological studies, the risk factors are evaluated mainly using hierarchical logistic regression (HLR) models that are based on conceptual models of the study. However, these models are limited to conditional risk estimation for the outcome variable using single or multiple explanatory variables (Kastorini et al., 2015) and cannot be a robust method of estimation in aforementioned complicated conceptual models. This study argues that the interwoven and bifurcation potential of relationships can be estimated through structural equation modeling (SEM) rather than using traditional logit function. However, the binary outcome variable could lead to the presence of a non-linear relationship between predictors and outcome variables that needs special consideration in SEM before estimation. There are numerous studies in the literature which highlighted non-linear relationships between many risk factors like income and CVDs (Havranek et al., 2015), income with hypertension and cholesterol abnormality (Rehkopf, Krieger, Coull, \& Berkman, 2010), MS and sleep (Fan et al., 2020) and smoking levels and CVDs (Lim et al., 2012). Therefore, the study aims to estimate linear and non-linear pathways of complex model based on background, modifiable factors and CVDs and isolate the mediation impact of potential mediators especially MS. Further, this study is trying to overcome the under generalization problem arises due to a lack of a unified definition of MS by using the number of metabolic syndrome abnormalities (MSA) instead of MS.

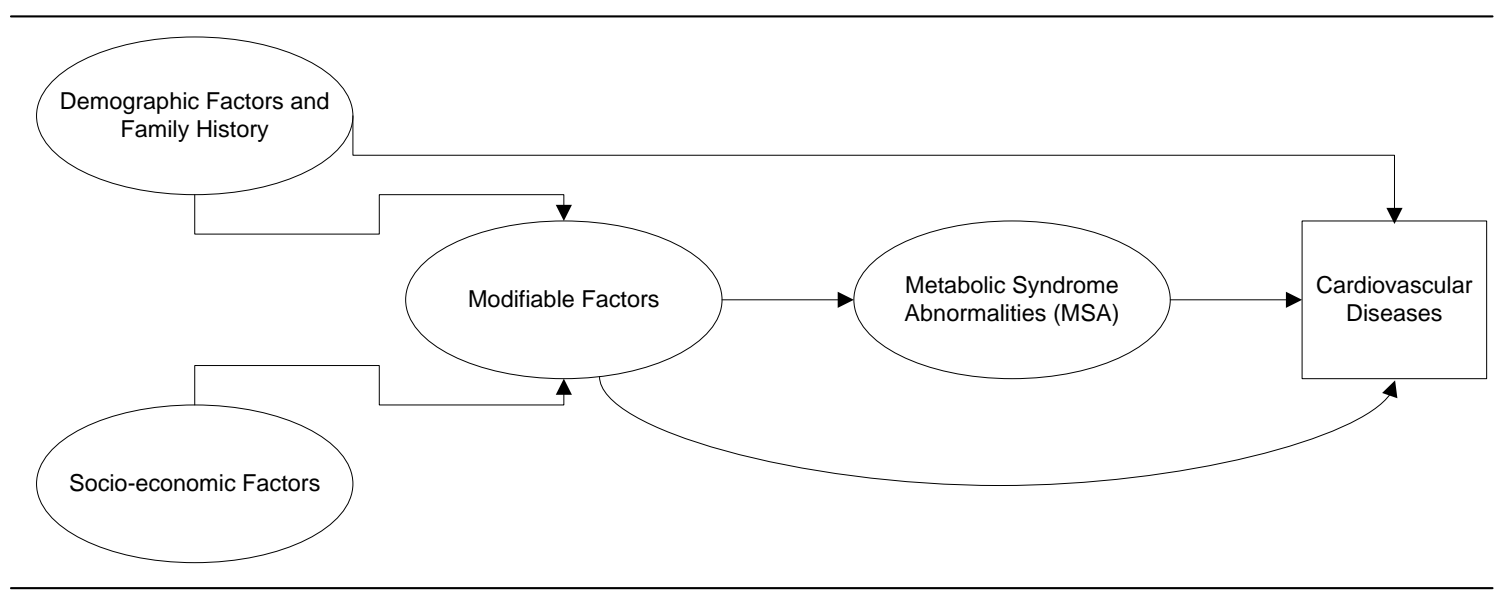

Figure 1: Conceptual model of associated factors of cardiovascular diseases

\section{Material and methods}


In this study, most populated and diversified province (Punjab) of Pakistan was selected as the sampled population of the study. This province is economically established and has comparatively good health facilities (Statistics, 2017). The residents of other provinces are usually travelled to this region of the country for the treatment of chronic and complex diseases. Further, it is consisting of more than 50\% population of the country. The selected hospital is the largest cardiovascular hospital in Pakistan and provides services across the country (https://pic.punjab.gov.pk/Statistics). Therefore, it would be possible to generalize the findings of the study to the country. This was a case-control study, with individuals matched by sex. Cases and controls were diagnosed and declared by certified duty physicians. Those persons, who had age above 30 years, having at least one CVDs (except congenital diseases), were considered as cases for the current study. On the other side, the person who did not show any clinical sign and symptom of CVDs were the controls. Most importantly, this study focuses only on those cases who get any problem of CVDs first time in their lifespan. This study was conducted from September 2018 to February 2019 in Punjab Institute of Cardiology (PIC) hospital, Lahore. Cases of the study were selected through systematic sampling. Further, 234 consecutive cases who registered in PIC hospital and fulfilled the above-mentioned inclusion criteria were chosen systematically for this study. The study was approved by the ethical review committee of the PIC hospital, Lahore. The patients who had, age less than 30 years, any history of CVDs events, congenital heart problems and recently changed dietary habits, were excluded from the study. For the patients who could not answer due to severe condition, speech disorder, unconsciousness and such, their information was retrieved from the first-degree relative or attendants (preferably lived in the same house and aware about the medical history and dietary habits of the patients). Further, 234 hospital-based controls were selected from the attendants of patients available in the same hospital by the investigators. The selection of hospital-based controls can produce valuable findings by reducing biases and improving the quality of information as compared to population-based controls ( $\mathrm{Li} \& \mathrm{Zhang}, 2011)$. The data was collected by the researchers with the help of the nursing staff. The sample size was computed through Warp partial least squares (PLS) software version 6.0. The Warp PLS technique needs a sample size usually based on the power of test and level of significance. A sample of 468 subjects with a power of 0.80 and $5 \%$ level of significance was estimated. This total sample was divided in equal proportion of cases and controls to follow 1:1 matching ratio. Out of 468 filled questionnaires, only 8 questionnaires were excluded from the study due to incomplete information on the mediating variable of the study.

The detailed medical history of all participants (cases and controls) was recorded on the questionnaire. It consisted of background factors (age, gender and area, family history (FH) of CVDs), SE, modifiable factors and components of MS. FH of CVDs was defined as any cardiovascular event among any first degree relatives (that is, biological parents and full siblings) of the participants (Song et al., 2019). Level of education of the participants was measured by years of schooling. Financial status was measured through subjective financial wellbeing (SFW) status suggested in the literature to avoid misinformation and potential refusal of the participants on the direct question about income. (Arber, Fenn, \& Meadows, 2014; Singh-Manoux, Marmot, \& Adler, 2005). SFW can measure the level of difficulty faced by individuals in managing income and household expenses. Occupation of the participant was measured in terms of the demand in physical activity (PA) as discussed in recent CVDs studies (Kastorini et al., 2015; Wareham et al., 2003). International physical activity questionnaire (IPAQ) was used to assess the PA level in the study participants as discussed by Papathanasiou et al. (2009). Sleep satisfaction (SS) of the participants were measured by asking about their level of satisfaction regarding sleep that ranges from very dissatisfied "1" to very satisfied "5". Further, selfreported subjective stress (SSS) was assessed also using a 5-unit scale that ranges from "not at all stressful" to "very stressful" (Godwin et al., 2008). Smoking status was categorized into three categories which were current smoker, former smoker and non-smokers as used in the recent literature (Kastorini et al., 2015). However, alcohol consumption was not considered in this study due to very low consumption in the Pakistani population (Organization, 2018) as it is religiously, culturally and legally prohibited activity.

Dietary habits were evaluated using a modified food frequency questionnaire (FFQ) developed after careful consideration of Pakistani foods groups and items (M. R. Sajid, Muhammad, Zakaria, \& Shahbaz, 2019). The modified FFQ was confirmed using 12 food groups out of 13 food groups which were further classified into two main groups; positive dietary habits (PDH) and negative dietary habits (NDH). Psychometric properties of the modified FFQ were tested and found reliable with 0.905 and 0.879 reliability coefficient for positive and negative dietary habits respectively. Exploratory factor analysis (EFA) explored these two groups with an acceptable level of explained variation. However, in the structural model, only NDH score was used to overcome the probable problem of multicollinearity (a basic assumption in partial least squares approach) further literature also suggested that NDH are highly associated with morbidity and mortality of diseases as compared to PDH (Menotti et al., 2012). 
Metabolic syndrome (MS) status is based on following five criteria or abnormalities: 1) abdominal obesity assessed through waist circumference; 2) elevated triglycerides; 3) decreased high-density lipoprotein (HDL) cholesterol; 4) high blood pressure; and 5) increased fasting glucose (Panel, 2001). Due to different definitions (Alberti et al., 2009; Federation, 2006; Panel, 2001) about the classification of MS in the literature researchers used the presence of number of abnormalities (as per NCEP-III guidelines for Asian people) in cases and controls which ranges from $0=$ absence of all abnormalities, to, $5=$ presence of all abnormalities of MS. This approach would increase the generalizability of the study findings rather than following particular criteria of MS. The term MSA was also used in the literature (Révész, Milaneschi, Verhoeven, Lin, \& Penninx, 2015).

\subsection{Statistical analysis}

Quantitative and qualitative variables were described in terms of mean \pm standard deviation (SD) and frequency distribution, respectively. Proportion test was implied to see weather case and control groups differ significantly on the single category of qualitative variables or not. Independent sample t-test was also used to evaluate the significant difference between cases and controls for quantitative variables after assessing normality assumption through Kolmogorov Smirnove test. The statistical significance was reported at 0.05 level of significance with the two-sided hypothesis in the study. SPSS 21.0 was used for these descriptive statistics. All characteristics in the model were observed variables therefore path analyses were used to test the interrelationships of factors of CVDs.

\subsection{Path analysis and Warp PLS}

In the proposed model given in Figure 1, the possible risk factors of CVDs were linked through multiple ways; directly and indirectly. Path analyses based on SEM was used to estimate the true influence of factors in different relational paths. It is the second generation multivariate technique and extension of regression analysis that estimates the path coefficients through simultaneous equations approach which minimizes the biasness in the findings (Kock, 2017). The general SEM model is given in equation (1).

$$
\left[\begin{array}{c}
y_{1} \\
y_{2} \\
\cdot \\
\cdot \\
\cdot \\
y_{p}
\end{array}\right]=\left[\begin{array}{cccc}
0 & \beta_{12} & \cdots & \beta_{1 p} \\
\beta_{21} & 0 & \cdots & \beta_{2 p} \\
\cdot & \cdot & \cdot & \cdot \\
\cdot & \cdot & \cdot & \cdot \\
\cdot & \cdot & \cdot & \cdot \\
\beta_{p 1} & \beta_{p 2} & \cdots & 0
\end{array}\right]\left[\begin{array}{c}
y_{1} \\
y_{2} \\
\cdot \\
\cdot \\
\cdot \\
y_{p}
\end{array}\right]+\left[\begin{array}{cccc}
\gamma_{11} & \gamma_{12} & \cdots & \gamma_{1 q} \\
\gamma_{21} & \gamma_{22} & \cdots & \gamma_{2 q} \\
\cdot & \cdot & \cdot & \cdot \\
\cdot & \cdot & \cdot & \cdot \\
\cdot & \cdot & \cdot & \cdot \\
\gamma_{p 1} & \gamma_{p 2} & \cdots & \gamma_{p q}
\end{array}\right]\left[\begin{array}{c}
x_{1} \\
x_{2} \\
\cdot \\
\cdot \\
\cdot \\
x_{q}
\end{array}\right]+\left[\begin{array}{c}
\zeta_{1} \\
\zeta_{2} \\
\cdot \\
\cdot \\
\zeta_{p}
\end{array}\right]
$$

where $y_{i}$ be the dependent variable and $x_{j}$ be an independent variable where $i=1,2, \ldots p, j=1,2, \ldots q$. The regression coefficient is denoted by $\beta_{i i}$ for variable $y_{i}$ with another variable $y_{i}$, where $i \neq i$. Similarly, $\gamma_{i j}$ be a regression coefficient of $y_{i}$ on variable $x_{i}$. The error vector is denoted by $\zeta_{i}$. Equation (1) shows that there are $p$ simultaneous equations (paths) in this model. The analysis was performed using Warp PLS 6.0 software due to the binary outcome variable of the model. This software is based on partial least squares (PLS) approach which is well suited to capture linear as well as non-linear simultaneous relationships of real phenomenon (Kock, 2015). Further, no assumption of normality of data and the large sample size is required as suggested in covariance-based SEM and other multivariate statistical techniques (Urbach \& Ahlemann, 2010). In this study, Warp 3 algorithm was used as it performs well in case of the main binary endogenous variable and considered sigmoid-curve for the estimation of associated paths (Kock, 2015). Warp 3 algorithm transforms (or warps) the explanatory variables scores or values which have U-curves first derivatives. In the binary outcome endogenous variable, S-shaped or sigmoid curve is possible which can be observed as a combination of two U-shaped curves, where one is inverted. The analysis was performed with 500 resamples through bootstrapping method due to the complex model of study as suggested in the literature (Kock, 2017). All factors of the model were observed variables, nonetheless, Warp PLS used the oval shape for all types of variables. The unobserved heterogeneity in the relationships of path analysis was also studied. This heterogeneity affects the overall coefficient (global coefficient) in term of over/underestimation. Best fitting curves and segments of main relationships for all paths were drawn considering multivariate relationships to identify nonlinear relationships in the path analysis. Further, Warp PLS used different approaches to evaluate the mediation effect, however, estimation of indirect effects approach was used that allows estimation of multiple mediating effects. This mediation analysis uses a resampling procedure and minimizes the errors in the estimation procedure. 


\section{Results}

\subsection{Basic characteristics of study participants}

Descriptive statistics of the study factors are presented in Table 1 . The average age of the cases was slightly higher than controls of study. Out of 460, $312(67.8 \%)$ participants of the study were males. Family history of CVDs was more than double in cases $(35.6 \%$ ) as compared to controls (with p-value $=<0.001$ ). Controls were having significantly higher average years of schooling than cases of study ( $\mathrm{t}$-value $=-4.49$, $\mathrm{p}$-value $<0.001)$. Almost half $(53 \%)$ of the cases have sedentary occupation while $24 \%$ had been involved in heavy manual work. The frequency distribution of occupation reflected U-shaped distribution in the cases dataset. In response to SFW, $31 \%$ cases were facing severe difficulty in managing the day to day expenses. However, no significant difference was observed in cases and controls proportions in different categories of SFW. Prevalence of current smoking in cases was 38.2\% which was almost 2.2 times than controls of study. Only 4 females were smokers in the study (not reported in Table 1). The average score of NDH in cases (3.27) was significantly higher than controls (1.92) of study with t-value $=20.12$. Approximately half of the cases were having low PA profile (inactive) which was twice as compared to controls (24.4\%). MSA was more prevalent in cases and no case was found in the study without at least one MSA while $10 \%$ controls did not show any MSA.

Table 1: Descriptive statistics of cases and controls according to variables of the model

\begin{tabular}{|c|c|c|c|c|}
\hline No. & Characteristics & CVDs Patients & CVDs Control & p-value \\
\hline 1 & Age (in years) & $50.11 \pm 11.15$ & $48.30 \pm 10.84$ & 0.078 \\
\hline 2 & Male & $156(67.8)$ & $156(67.8)$ & 1.000 \\
\hline 3 & Family history of CVDs & $82(35.6)$ & 36 (15.6) & $<0.001 * *$ \\
\hline 4 & Urban area & $157(68.3)$ & $111(48.3)$ & $<0.001 * *$ \\
\hline 5 & Education (years) & $8 \pm 5.20$ & $10 \pm 4.3$ & $<0.001 * *$ \\
\hline \multirow[t]{5}{*}{6} & Occupation & & & \\
\hline & Sedentary occupation & $122(53.0)$ & $49(21.3)$ & $<0.001 * *$ \\
\hline & Standing occupation & $23(10.0)$ & $64(27.8)$ & $<0.001 * *$ \\
\hline & Physical work & $30(13.0)$ & $80(35.0)$ & $<0.001 * *$ \\
\hline & Heavy manual work & $55(24.0)$ & $37(16.1)$ & $<0.035^{*}$ \\
\hline \multirow[t]{6}{*}{7} & Subjective financial wellbeing (SFW) & & & \\
\hline & Manage with great difficulty & $71(31.0)$ & $53(23.0)$ & 0.058 \\
\hline & Manage with difficulty & $49(21.3)$ & $40(17.3)$ & 0.289 \\
\hline & Manage with some difficulty & $30(13.0)$ & $33(14.2)$ & 0.682 \\
\hline & Manage fairly easy & $49(21.3)$ & $63(27.4)$ & 0.128 \\
\hline & Manage very easily & $31(13.4)$ & $41(18.1)$ & 0.201 \\
\hline \multirow[t]{4}{*}{8} & Smoking Status & & & \\
\hline & Never smoker & $103(44.8)$ & $165(71.7)$ & $<0.001 * *$ \\
\hline & Former smoker & $39(17.0)$ & $25(10.9)$ & 0.058 \\
\hline & Current smoker & $88 \quad(38.2)$ & $40 \quad(17.4)$ & $<0.001 * *$ \\
\hline \multirow[t]{6}{*}{9} & Sleep satisfaction & & & \\
\hline & Very satisfied & $34(15.0)$ & $66 \quad(29.0)$ & $<0.001 * *$ \\
\hline & Satisfied & $99(43.0)$ & $106(46.0)$ & 0.512 \\
\hline & Somewhat satisfied & $24(10.5)$ & $21(9.0)$ & 0.6383 \\
\hline & Dissatisfied & $42(18.2)$ & $20(8.6)$ & $<0.001 * *$ \\
\hline & Very dissatisfied & $31(13.3)$ & $17(7.4)$ & $<0.032 *$ \\
\hline \multirow[t]{6}{*}{10} & Subjective self-reported stress & & & \\
\hline & Very stressful & $27(12.0)$ & $17(7.4)$ & 0.112 \\
\hline & Stressful & $33(14.0)$ & $22(9.6)$ & 0.114 \\
\hline & Sometimes stressful & $21(9.1)$ & $14(6.0)$ & 0.218 \\
\hline & Rarely stressful & $92(40.0)$ & $124(54.0)$ & $<0.001 * *$ \\
\hline & Not at all stressful & $39(16.9)$ & $53(23.0)$ & 0.103 \\
\hline \multirow{3}{*}{$\begin{array}{l}11 \\
12\end{array}$} & Negative dietary habits & $3.3 \pm 0.88$ & $1.92 \pm 0.51$ & $<0.001 * *$ \\
\hline & Physical activity level & & & \\
\hline & Low profile & $113(49.0)$ & $56 \quad(24.4)$ & $<0.001 * *$ \\
\hline
\end{tabular}




\begin{tabular}{cccc}
\hline $\begin{array}{c}\text { Moderate profile } \\
\text { High profile }\end{array}$ & $91(39.7)$ & $113(49.0)$ & $<0.040^{*}$ \\
$13 \quad 26(11.3)$ & $61(26.6)$ & $<0.001^{* *}$ \\
Metabolic syndrome abnormalities & & & \\
0 & $0(0.0)$ & $23(10.0)$ & $<0.001^{* *}$ \\
1 & $7(3.0)$ & $51(22.0)$ & $<0.001^{* *}$ \\
2 & $45(19.5)$ & $71(31.0)$ & $<0.001^{* *}$ \\
4 & $55(24.0)$ & $73(32.0)$ & 0.061 \\
5 & $65(28.3)$ & $10(4.0)$ & $<0.001^{* *}$ \\
& $58(25.2)$ & $2(1.0)$ & $<0.001^{* *}$ \\
\hline
\end{tabular}

* significant at $5 \%$ level of significance, ** significant at $1 \%$ level of significance

\subsection{Model fitness indices of path analyses}

Path analyses were performed on the structural model (Figure 2) derived from the conceptual model of study (Figure 1). It was hypothesized that demographic and SE factors influence modifiable factors which further affect CVDs outcome directly or indirectly through MSA. Besides, family history could also affect CVDs directly. Figure 2 was presented with the results of path analyses. The significant paths of the model were bold and all path coefficients mentioned in the exact center of each unidimensional arrow. All model fit-indices reported in Table 2, reflected the fitness of the model with data. These fit-indices are the depiction of model fitness for explaining the phenomenon. There are two important measures to evaluate the model; average path coefficients with their $\mathrm{R}^{2}$ value and assessment of multicollinearity assumption as suggested in the literature (Berglund, Lytsy, \& Westerling, 2013). Average path coefficients were 0.154 which was significant at 0.01 level of significance. Further, PLS approach is based on variation based model formulation, therefore, average $\mathrm{R}^{2}(0.217)$ was significant and average full collinearity VIF (AFVIF) which is an assessment of multicollinearity (1.507) was also less than the cut-off value $(<=5)$.

Table 2: Model fit and quality indices

\begin{tabular}{cccc}
\hline Sr. No. & Measures & $\begin{array}{c}\text { Estimated value } \\
(\text { significance })\end{array}$ & Evaluation criteria \\
\hline 1 & Average path coefficient (APC) & $0.154(\mathrm{p}<0.001)$ & $\mathrm{p}$-value should be less than 0.05. \\
2 & Average R-squared (ARS) & $0.217(\mathrm{p}<0.001)$ & $\mathrm{p}$-value should be less than 0.05. \\
3 & Average adjusted R-squared (AARS) & $0.208(\mathrm{p}<0.001)$ & $\mathrm{p}$-value should be less than 0.05. \\
4 & Average block VIF (AVIF) & 1.162 & acceptable if $<=5$ \\
5 & Average full collinearity VIF (AFVIF) & 1.507 & acceptable if $<=5$ \\
\hline
\end{tabular}

\subsection{Path analyses}

Findings of initial path analyses, which was performed without considering the mediation impact of MSA, (not reported in the Figure 2 due to reporting of mediation impact through direct paths) revealed that all modifiable factors including smoking $(\beta=0.160, \mathrm{p}<0.001)$, SSS $(\beta=0.138, \mathrm{p}=0.008)$, PA $(\beta=-0.358, \mathrm{p}<0.001)$, SS $(\beta=-0.272, \mathrm{p}$ $<0.001)$ and negative dietary habits $(\beta=0.157, \mathrm{p}=0.003)$ significantly associated directly with CVDs at given level of significance (5\%). High SS and high profile PA (with negative $\beta$ 's) were protective factors of CVDs. The final model was presented in Figure 2 and its results reported in Table 3 was formulated to assess the mediation impact of MSA and other modifiable factors. Self-reported subjective stress ( $\beta=0.163, p=0.009)$ would increase as age grows while SS $(\beta=-0.222, p<0.001)$ and PA $(\beta=-0.387, p<0.001)$ reduced. Males were associated with smoking $(\beta=$ $0.448, \mathrm{p}<0.001)$ and negative dietary habits $(\beta=0.114, \mathrm{p}<0.001)$. Family history also influenced the SS, SSS, PA and CVDs directly. However, FH had shown the highest influence on SSS $(\beta=0.212, p<0.001)$ as compared to other modifiable factors. The urban area was strongly associated with NDH $(\beta=0.202, p<0.001)$. SE factors (SFW, education and occupation) analysis showed that SFW was the dependent characteristic of education and occupation and further all SE factors showed a strong influence on the modifiable risk factors. All SE factors were strongly predicting the SSS like the majority of demographic factors. All modifiable factors (except PA, $\beta=-0.070, p>0.05$ ) were strongly associated with occupation. However, this PA was the strongest predictor $(\beta=-0.285, p<0.001)$ of MSA among all modifiable factors. Higher the profile of PA lower will be the MSA. Further, all modifiable risk 
factors except SSS were strongly related with MSA and found that SSS was also linked with other modifiable factors which were SS $(\beta=-0.186, p<0.001)$ and NDH $(\beta=0.206, p<0.001)$. Further, non-linear relationships were found between two pairs, age and NDH, and education and SSS. Therefore, linear segmentation of non-linear relationships was performed to estimate segment-wise estimates for identified linear segments. The global estimate of age and NDH was $(\beta=0.060, p=0.265$ given in Table 3$)$ and coefficients of linear segments were, $\beta=-0.06, p=0.10 ; \beta=0.04$, $\mathrm{p}=0.17 ; \beta=0.07, \mathrm{p}=0.06$ for age groups, $30-36,36-46,46-68$ respectively. The relationship became negative and steeper in later age groups (68+ years). Similarly, non-linear relationship of education and SSS was converted into linear segments which were, $\beta=-0.17, \mathrm{p}<0.01 ; \beta=0.13, \mathrm{p}<0.01 ; \beta=0.20, \mathrm{p}<0.01$ and $\beta=0.03, \mathrm{p}=0.27$ for $>$ 5, 5-8, 8-19 and 19+ years of education respectively. These mixed findings for these two pairs of relationships made the overall relationships as non-linear ones.

\subsection{Assessment of mediation through indirect effects}

The finalized model (Figure 2) also computed the mediating role of MSA. The direct arrows from modifiable factors to CVDs were the mediating path coefficients in the proposed relationships. Table 4 is the summary of the mediating paths and their corresponding empirical assessment. MSA played a mediating role for the relationship of all modifiable factors with CVDs except SSS at $5 \%$ level of significance. The MSA was the strongest mediator $(\beta=-0.109, \mathrm{p}<0.001)$ for the relationship between PA and CVDs. The insignificant role of MSA for SSS and CVDs motivated to explore some more alternative paths which were $\mathrm{SSS} \rightarrow \mathrm{SS} \rightarrow \mathrm{MSA}$ and $\mathrm{SS} \rightarrow \mathrm{NDH} \rightarrow \mathrm{MSA}$. Table 4 depicted that the relationship of SSS with MSA was mediated through SS $(\beta=-0.033$, p-value $=0.011)$ and NDH $(\beta=0.031, \mathrm{p}$-value $=0.012$ ). The role of MSA and two other modifiable factors as mediator was confirmed in the study through path analyses.

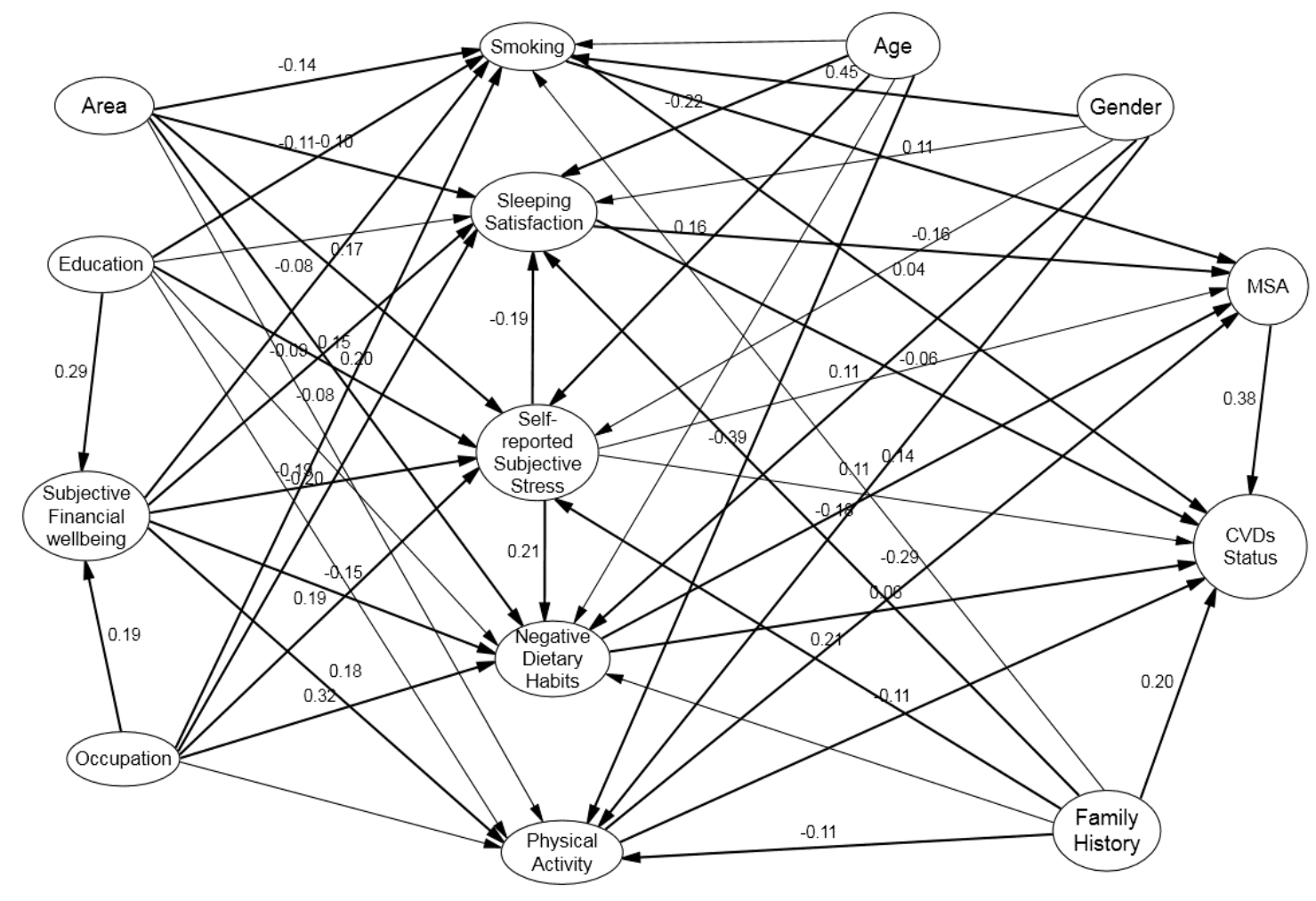

Figure 2: Path analyses for associated factors of CVDs

Table 3: Direct path coefficients of the path model 


\begin{tabular}{cccccc}
\hline Paths & $\begin{array}{c}\text { Path coefficients } \\
(\beta \text { 's })\end{array}$ & p-value & Paths & $\begin{array}{c}\text { Path coefficients } \\
(\beta \text { 's })\end{array}$ & p-value \\
\hline Age $\rightarrow$ SS & -0.222 & $<0.001$ & Education $\rightarrow$ SS & 0.069 & 0.113 \\
Age $\rightarrow$ Smoking & 0.061 & 0.096 & Education $\rightarrow$ Smoking & -0.108 & 0.007 \\
Age $\rightarrow$ SSS & 0.163 & 0.009 & Education $\rightarrow$ SSS & 0.147 & $<0.001$ \\
Age $\rightarrow$ NDH & 0.060 & 0.265 & Education $\rightarrow$ NDH & -0.062 & 0.091 \\
Age $\rightarrow$ PA & -0.387 & $<0.001$ & Education $\rightarrow$ PA & 0.059 & 0.100 \\
Gender $\rightarrow$ SS & -0.068 & 0.064 & Education $\rightarrow$ SFW & 0.288 & $<0.001$ \\
Gender $\rightarrow$ Smoking & 0.448 & $<0.001$ & Occupation $\rightarrow$ SS & -0.198 & $<0.001$ \\
Gender $\rightarrow$ SSS & -0.059 & 0.103 & Occupation $\rightarrow$ Smoking & -0.081 & 0.045 \\
Gender $\rightarrow$ NDH & 0.114 & 0.004 & Occupation $\rightarrow$ SSS & 0.192 & 0.002 \\
Gender $\rightarrow$ PA & 0.108 & 0.034 & Occupation $\rightarrow$ NDH & -0.322 & $<0.001$ \\
FH $\rightarrow$ SS & -0.175 & $<0.001$ & Occupation $\rightarrow$ PA & -0.070 & 0.079 \\
FH $\rightarrow$ Smoking & 0.056 & 0.111 & Occupation $\rightarrow$ SFW & 0.189 & $<0.001$ \\
FH $\rightarrow$ SSS & 0.212 & $<0.001$ & SFW $\rightarrow$ SS & -0.089 & 0.021 \\
FH $\rightarrow$ NDH & -0.019 & 0.307 & SFW $\rightarrow$ Smoking & -0.075 & 0.048 \\
FH $\rightarrow$ PA & -0.103 & 0.016 & SFW $\rightarrow$ SSS & -0.188 & $<0.001$ \\
FH $\rightarrow$ CVDs & 0.203 & $<0.001$ & SFW $\rightarrow$ NDH & 0.152 & 0.043 \\
Area $\rightarrow$ SS & -0.104 & 0.041 & SFW $\rightarrow$ PA & 0.183 & $<0.001$ \\
Area $\rightarrow$ Smoking & -0.142 & 0.008 & SS $\rightarrow$ MSA & -0.159 & $<0.001$ \\
Area $\rightarrow$ SSS & 0.172 & $<0.001$ & Smoking $\rightarrow$ MSA & 0.112 & 0.048 \\
Area $\rightarrow$ NDH & 0.202 & $<0.001$ & SSS $\rightarrow$ MSA & 0.096 & 0.078 \\
Area $\rightarrow$ PA & 0.046 & 0.219 & NDH $\rightarrow$ MSA & 0.135 & 0.022 \\
& & & PA $\rightarrow$ MSA & -0.285 & $<0.001$ \\
& & SSS $\rightarrow$ SS & -0.186 & $<0.001$ \\
& & SSS $\rightarrow$ NDH & 0.206 & $<0.001$ \\
& & MSA $\rightarrow$ CVDS & 0.383 & $<0.001$ \\
\hline
\end{tabular}

Table 4: Assessment of the mediation effect

\begin{tabular}{cccc}
\hline Paths & Path coefficients $(\beta$ 's $)$ & p-value & Results \\
\hline SS $\rightarrow$ MSA $\rightarrow$ CVDs & -0.061 & 0.003 & Supported \\
Smoking $\rightarrow$ MSA $\rightarrow$ CVDs & 0.041 & 0.046 & Supported \\
SSS $\rightarrow$ MSA $\rightarrow$ CVDs & 0.037 & 0.081 & Not supported \\
NDH $\rightarrow$ MSA $\rightarrow$ CVDs & 0.052 & 0.027 & Supported \\
PA $\rightarrow$ MSA $\rightarrow$ CVDs & -0.109 & $<0.001$ & Supported \\
SSS $\rightarrow$ SS $\rightarrow$ MSA* & -0.033 & 0.011 & Supported \\
SSS $\rightarrow$ NDH $\rightarrow$ MSA* & 0.031 & 0.012 & Supported \\
\hline
\end{tabular}

*Not displayed in model figure 2 .

\section{Discussions}

Present work supported our propositions about the role of MSA and some other modifiable factors in the causal mechanism of CVDs that initiated from demographic and SE factors. The SE factors, education and occupation were strongly related to newly suggested SFW (a proxy of income). However, the role of each factor was diverse in the model. The significant enormity of CVDs risk has been split through MSA and transmit to CVDs. The background factors showed a strong influence to adopt certain modifiable or lifestyle-related practices that would decide the prevalence of MSA and cardiac health. Further, identification of non-linear paths would help in defining the pathways with deeper level. The identified mediation role of MSA and some modifiable factors will enable the policymakers and practitioners to understand the various routes of risk transmission from different layers of risk factors to CVDs. These novel pathways of risk flow would help in defining and identifying the risk group of the population that leads to set target-oriented strategies for the CVDs reduction. The path analyses empirically tested various routes of risk transmission using Warp 3 algorithm that could not be possible through traditional probability models. This study is the first study of its nature which covers maximum types of risk factors of CVDs and MSA as mediator. 
Smoking provided the linking path for the first pathway derived from the structural model. We found that males, people from the rural area, low education and SFW and doing heavy manual work increases the likelihood of smoking and consistent with the literature (A. L. Association, 2012; Organization, 2018). In contrary, the role of age and FH were found insignificant in smoking. Usually, the age-wise prevalence of smoking is decreased especially from middle-aged to elder adults (Scollo \& Winstanley, 2016). However, in present work, all participants of the study had no previous exposure of CVDs event (in cases) and sign and symptoms (in controls) which might urge them for the continuation of their smoking practices. On the other side, people process familial and behavioral factors separately that could decrease the impact of FH (Akhuemonkhan \& Lazo, 2017). In the pathway, now smoking can influence the CVDs directly or indirectly through MSA. The risk of MS and all its abnormalities is maximum in the current smoker and lowest in non-smokers (Chen et al., 2008) that corroborates our indirect findings. The mediating path (Smoking $\rightarrow$ MSA $\rightarrow$ CVDs) also got a significant amount of impact and confirmed this alternative route. This first pathway indicates poor and less educated rural males should be focused on smoking preventive strategies to reduce the burden of CVDs. Further, smokers can delay their CVDs risk by reducing the number of MSA especially by changing their lifestyles.

Present work found that advanced age, urbanized settings, positive FH, heavy manual work and low SFW cause low sleep satisfaction (SS) and validate previous literature. (Grandner et al., 2010; Greer, Sui, Maslow, Greer, \& Blair, 2015; Lavoie, Zeidler, \& Martin, 2018; Olsen, Nicholls, \& Mitchell, 2019; Patel, Grandner, Xie, Branas, \& Gooneratne, 2010). In contrast, highly educated people might have better awareness about sleep quality but involvement in a sedentary occupation leads to sedentary behaviour which reduces the required PA level (Thivel et al., 2018) and could engulf the impact of education on SS. Moreover, an approximately equal proportion of males and females complaining about low SS in present work. The dominating smoking habits in males and low PA in females could be the possible reasons for sleep dissatisfaction (Quan et al., 2016) that might create equal chances of sleep complainants in both genders. This pathway is also associated with the pathway of stress. Sleep quality and quantity both are associated with the normal physiology of the body and abnormalities cause metabolic syndrome. The literature highlighted the significant relationship of low-quality self-reported sleep (Hung et al., 2013) and less duration of sleep than normal (6-8 hours) with MS and its components that lead to CVDs (Wu et al., 2012). The sleep strategies can be designed especially for poor people and urban residents.

Frequency of SSS varies in different demographic and socio-economic factors. Advanced ages, urbanized settings, positive FH, high education and sedentary occupation and low SFW were reasons of SSS in the study (Cappelletti, Kreuter, Boyum, \& Thompson, 2015; Genin et al., 2018; Hernández, Pasupuleti, Deshpande, Bernabé-Ortiz, \& Miranda, 2012; Osmanovic-Thunström, Mossello, Åkerstedt, Fratiglioni, \& Wang, 2015; Song et al., 2019). On contrary to the literature we found an insignificant relationship between gender and SSS. It is might be due to the substantial proportion of sample of the study was in the age range of 40-47 years which is most stressful generation irrespective of the gender (A. P. Association, 2012). Surprisingly, MSA did not play a mediating role for SSS to CVDs. Further exploration reflected that the impact of SSS on MSA was transmitted through two other modifiable factors; SS and NDH. People in high SSS may have low SS and poor selection of foods (Barrington, Beresford, McGregor, \& White, 2014; Patel et al., 2010). SSS strengthen the role of SS and NDH in their paths and enhances predictive strength for CVDs. This novel pathway guides that stress has a multidimensional impact in CVDs phenomenon and should be considered while designing controlling strategies. Further, non-linear path between education and SSS should also kept in mind while identifying the risky individuals as well.

Food choices are largely affected through demographic, SE and other modifiable factors. Similar to SS, NDH can also receive the strength from SSS to predict MSA and CVDs. we found that NDH were widespread in males, urban area, people with better SFW and heavy manual work as discussed in the literature (Leblanc et al., 2015; Misra \& Khurana, 2009). However, the insignificant influence of age on NDH was also found due to inconsistent relationship (non-linear relationship) in different age groups. Education can reduce the NDH surge in society but LMIC need to work hard to achieve required levels. This insignificant relationship can be possible because all participants of the study had no previous exposure of CVDs event (in cases) and sign and symptoms (in controls) which might lead to the continuation of their routine dietary practices. Further, the insignificant impact of FH on the adaptation of NDH reflected that people usually process familial and behavioral factors separately (Akhuemonkhan \& Lazo, 2017). In this pathway, NDH which are usually consisted of intake of saturated fats, usage of red meat, sweetened and processed foods 
increase the MSA (Becerra-Tomás et al., 2016) that leads to CVDs. Present work provided a significant mediating role of MSA in the relationship of NDH and CVDs. This pathway indicates that by educating the people especially urban males, about the quality of foods, the incidence of MSA and CVDs can be delayed.

Physical activity provided a protective pathway to reduce the risk of CVDs. Physically active people can significantly reduce the risk of CVDs and MSA. We found that physical activities were reduced with increase in age, less prevalent in females and people with positive FH but high in people with better SFW. These findings also supported by literature (Bauman et al., 2009; Greer et al., 2015; Organization, 2018). However, the role of education, occupation, urban settings were found insignificant. People with better education might have better knowledge about the benefits of physical activities but their high education usually translates into good jobs and better income activities that lead to sedentary occupation and behaviour. This situation might decrease the significant impact of education and make it insignificant in the present work. We also found insignificant relationship between occupation and PA which might be due to less involvement of heavy manual workers and sedentary professionals in health-related physical activities. Further, rural/urban spectrum might behave differently in LMIC as compared to higher-income countries. In LMIC, low PA is a usual negative outcome of urban areas due to busy routine for income-generating activities while their rural counterparts do not have sufficient awareness and facilities regarding health-related physical activities (Janjua, Mahmood, Bhatti, \& Khan, 2015). We found that MSA was the strongest mediator for PA. High physical activities can slacken the potential risk of hypertension, abnormal glucose fasting level, non-normal lipid profile and insulin resistance, obesity and ultimately reduce the risk of CVDs (Alberti et al., 2009Alberti et al., 2009; Grundy, 2016). This pathway is most promising for the reduction of CVDs risk in the population. Female population, sedentary professionals and heavy manual workers should be the target group for awareness programs.

\section{Conclusion}

This study concludes that demographic and SE factors are the strong determinants of modifiable risk factors and CVDs. However, the etiology of CVDs is not simple due to multiple pathways created by the interplay of these factors. The path model estimated through Warp algorithm empirically tested that risk of CVDs is mainly associated with modifiable risk factors which individually driven by demographic and SE characteristics of individuals. Further, the MSA significantly mediating the majority of pathways and gaining importance in this CVDs causal mechanism. Warp partial least square estimated the hypothetical pathways which are very much aligned with the literature and could not be evaluated through traditional HLR. This study would help the practitioners to design multiple strategies for the reduction of CVDs by following identified direct and indirect pathways.

\section{Contribution of the study}

For the practitioners, this study provides a first comprehensive multilayer model that bridges various types of risk factors of CVDs. The novel pathways which are tested through second-generation statistical technique would highlight the probable routes of risk transmission of CVDs. Further, evidence regarding the novel role of MSA is also provided in this study. This issue-based model can be replicated in the exploration of other complex diseases, especially with possible non-linear relationships.

This study is an addition in the limited literature of simultaneous modeling of binary endogenous variables that is beyond the traditional logistic regression model. It provides a reference for modeling of complex diseases with linear and non-linear relationships. Further, it would highlight the utility of Warp partial least square algorithms that extend the usage of SEM especially in the presence of multiple mediators.

\section{References}

1. Akhuemonkhan, E., \& Lazo, M. (2017). Association between family history of diabetes and cardiovascular disease and lifestyle risk factors in the United States population: the 2009-2012 National Health and Nutrition Examination Survey. Preventive Medicine, 96, 129-134.

2. Alberti, K., Eckel, R. H., Grundy, S. M., Zimmet, P. Z., Cleeman, J. I., Donato, K. A., . . S Smith Jr, S. C. (2009). Harmonizing the metabolic syndrome: a joint interim statement of the international diabetes federation task force on epidemiology and prevention; national heart, lung, and blood 
institute; American heart association; world heart federation; international atherosclerosis society; and international association for the study of obesity. Circulation, 120(16), 1640-1645.

3. Arber, S., Fenn, K., \& Meadows, R. (2014). Subjective financial well-being, income and health inequalities in mid and later life in Britain. Social Science and Medicine, 100, 12-20.

4. Association, A. L. (2012). Cutting tobacco's rural roots: Tobacco use in rural communities Disparities in Lung Health Series.

5. Association, A. P. (2012). Stress by generations (pp. 19-21).

6. Barrington, W. E., Beresford, S. A., McGregor, B. A., \& White, E. (2014). Perceived stress and eating behaviors by sex, obesity status, and stress vulnerability: findings from the vitamins and lifestyle (VITAL) study. Journal of the Academy of Nutrition and Dietetics, 114(11), 1791-1799.

7. Bauman, A., Bull, F., Chey, T., Craig, C. L., Ainsworth, B. E., Sallis, J. F., . . Pratt, M. (2009). The international prevalence study on physical activity: results from 20 countries. International journal of behavioral nutrition and physical activity, 6(1), 21.

8. Becerra-Tomás, N., Babio, N., Martínez-González, M. Á., Corella, D., Estruch, R., Ros, E., . . Lamuela-Raventós, R. M. (2016). Replacing red meat and processed red meat for white meat, fish, legumes or eggs is associated with lower risk of incidence of metabolic syndrome. Clinical Nutrition, 35(6), 1442-1449.

9. Berglund, E., Lytsy, P., \& Westerling, R. (2013). Adherence to and beliefs in lipid-lowering medical treatments: a structural equation modeling approach including the necessity-concern framework. Patient Education and Counseling, 91(1), 105-112.

10. Braveman, P., \& Gottlieb, L. (2014). The social determinants of health: it's time to consider the causes of the causes. Public Health Reports, 129(1_suppl2), 19-31.

11. Cappelletti, E. R., Kreuter, M. W., Boyum, S., \& Thompson, T. (2015). Basic needs, stress and the effects of tailored health communication in vulnerable populations. Health Education Research, 30(4), 591-598.

12. Cappuccio, F. P., Cooper, D., D'elia, L., Strazzullo, P., \& Miller, M. A. (2011). Sleep duration predicts cardiovascular outcomes: a systematic review and meta-analysis of prospective studies. European Heart Journal, 32(12), 1484-1492.

13. Chen, C.-C., Li, T.-C., Chang, P.-C., Liu, C.-S., Lin, W.-Y., Wu, M.-T., . . . Lin, C.-C. (2008). Association among cigarette smoking, metabolic syndrome, and its individual components: the metabolic syndrome study in Taiwan. Metabolism: Clinical and Experimental, 57(4), 544-548.

14. Fan, L., Hao, Z., Gao, L., Qi, M., Feng, S., \& Zhou, G. (2020). Non-linear relationship between sleep duration and metabolic syndrome: A population-based study. Medicine, 99(2), e18753.

15. Federation, I. D. (2006). The IDF consensus worldwide definition of the metabolic syndrome IDF Communications (pp. 1-23).

16. Genin, P., Dessenne, P., Finaud, J., Pereira, B., Dutheil, F., Thivel, D., \& Duclos, M. (2018). Effect of Work-Related Sedentary Time on Overall Health Profile in Active vs. Inactive Office Workers. Frontiers in public health, 6, 279. doi: 10.3389/fpubh.2018.00279

17. Godwin, M., Streight, S., Dyachuk, E., van Den Hooven, E. C., Ploemacher, J., Seguin, R., \& Cuthbertson, S. (2008). Testing the simple lifestyle indicator questionnaire: initial psychometric study. Canadian Family Physician, 54(1), 76-77.

18. Grandner, M. A., Patel, N. P., Gehrman, P. R., Xie, D., Sha, D., Weaver, T., \& Gooneratne, N. (2010). Who gets the best sleep? Ethnic and socioeconomic factors related to sleep complaints. Sleep medicine, 11(5), 470-478.

19. Greer, A. E., Sui, X., Maslow, A. L., Greer, B. K., \& Blair, S. N. (2015). The effects of sedentary behavior on metabolic syndrome independent of physical activity and cardiorespiratory fitness. Journal of Physical Activity and Health, 12(1), 68-73.

20. Grundy, S. M. (2016). Metabolic syndrome update. Trends in Cardiovascular Medicine, 26(4), 364373.

21. Havranek, E. P., Mujahid, M. S., Barr, D. A., Blair, I. V., Cohen, M. S., Cruz-Flores, S., . . Lockwood, D. W. (2015). Social determinants of risk and outcomes for cardiovascular disease: a scientific statement from the American Heart Association. Circulation, 132(9), 873-898.

22. Hernández, A. V., Pasupuleti, V., Deshpande, A., Bernabé-Ortiz, A., \& Miranda, J. J. (2012). Effect of rural-to-urban within-country migration on cardiovascular risk factors in low-and middle-income countries: a systematic review. Heart, 98(3), 185-194. 
23. Hung, H.-C., Yang, Y.-C., Ou, H.-Y., Wu, J.-S., Lu, F.-H., \& Chang, C.-J. (2013). The association between self-reported sleep quality and metabolic syndrome. PloS one, 8(1), e54304.

24. Janjua, N. Z., Mahmood, B., Bhatti, J. A., \& Khan, M. I. (2015). Association of household and community socioeconomic position and urbanicity with underweight and overweight among women in Pakistan. PloS one, 10(4), e0122314.

25. Kastorini, C.-M., Milionis, H. J., Georgousopoulou, E., Kalantzi, K., Nikolaou, V., Vemmos, K. N., ... Panagiotakos, D. B. (2015). Defining the path between social and economic factors, clinical and lifestyle determinants, and cardiovascular disease. Global heart, 10(4), 255-263.

26. Kock, N. (2015). PLS-based SEM algorithms: The good neighbor assumption, collinearity, and nonlinearity. Information Management and Business Review, 7(2), 113-130.

27. Kock, N. (2017). WarpPLS user manual: Version 6.0. ScriptWarp Systems: Laredo, TX, USA.

28. Lavoie, C. J., Zeidler, M. R., \& Martin, J. L. (2018). Sleep and aging. Sleep Science and Practice, 2(1), 3. doi: 10.1186/s41606-018-0021-3

29. Leblanc, V., Hudon, A.-M., Royer, M.-M., Corneau, L., Dodin, S., Bégin, C., \& Lemieux, S. (2015). Differences between men and women in dietary intakes and metabolic profile in response to a 12week nutritional intervention promoting the Mediterranean diet. Journal of nutritional science, 4 , e13.

30. Li, L., \& Zhang, M. (2011). Population versus hospital controls for case-control studies on cancers in Chinese hospitals. BMC medical research methodology, 11(1), 167.

31. Lim, S. S., Vos, T., Flaxman, A. D., Danaei, G., Shibuya, K., Adair-Rohani, H., . . Andrews, K. G. (2012). A comparative risk assessment of burden of disease and injury attributable to 67 risk factors and risk factor clusters in 21 regions, 1990-2010: a systematic analysis for the Global Burden of Disease Study 2010. The Lancet, 380(9859), 2224-2260.

32. Menotti, A., Alberti-Fidanza, A., Fidanza, F., Lanti, M., \& Fruttini, D. (2012). Factor analysis in the identification of dietary patterns and their predictive role in morbid and fatal events. Public health nutrition, 15(7), 1232-1239.

33. Misra, A., \& Khurana, L. (2009). The metabolic syndrome in South Asians: epidemiology, determinants, and prevention. Metabolic syndrome and related disorders, 7(6), 497-514.

34. Muhammad, N., Sajid, M. R., Zakaria, R., \& Shahbaz, A. (2019). The mediating role of metabolic syndrome in cardiovascular diseases. AIP Conference Proceedings, 2138(1), 050020. doi: $10.1063 / 1.5121125$

35. Olsen, J. R., Nicholls, N., \& Mitchell, R. (2019). Are urban landscapes associated with reported life satisfaction and inequalities in life satisfaction at the city level? A cross-sectional study of 66 European cities. Social Science and Medicine, 226, 263-274.

36. Organization, W. H. (2018). Noncommunicable Diseases (NCD) Country Profiles, .

37. Osmanovic-Thunström, A., Mossello, E., Åkerstedt, T., Fratiglioni, L., \& Wang, H.-X. (2015). Do levels of perceived stress increase with increasing age after age 65? A population-based study. Age and Ageing, 44(5), 828-834.

38. Panel, E. (2001). Executive summary of the third report of the National Cholesterol Education Program (NCEP) expert panel on detection, evaluation, and treatment of high blood cholesterol in adults (adult treatment panel III) JAMA (Vol. 285, pp. 2486-2497).

39. Papathanasiou, G., Georgoudis, G., Papandreou, M., Spyropoulos, P., Georgakopoulos, D., Kalfakakou, V., \& Evangelou, A. (2009). Reliability measures of the short International Physical Activity Questionnaire (IPAQ) in Greek young adults. Hellenic J Cardiol, 50(4), 283-294.

40. Patel, N. P., Grandner, M. A., Xie, D., Branas, C. C., \& Gooneratne, N. (2010). " Sleep disparity" in the population: poor sleep quality is strongly associated with poverty and ethnicity. BMC public health, 10(1), 475.

41. Quan, S.-A., Li, Y.-C., Li, W.-J., Li, Y., Jeong, J.-Y., \& Kim, D.-H. (2016). Gender differences in sleep disturbance among elderly Koreans: Hallym Aging Study. Journal of Korean Medical Science, 31(11), 1689-1695.

42. Rehkopf, D. H., Krieger, N., Coull, B., \& Berkman, L. F. (2010). Biologic risk markers for coronary heart disease: nonlinear associations with income. Epidemiology, 38-46.

43. Révész, D., Milaneschi, Y., Verhoeven, J. E., Lin, J., \& Penninx, B. W. (2015). Longitudinal associations between metabolic syndrome components and telomere shortening. The Journal of Clinical Endocrinology \& Metabolism, 100(8), 3050-3059. 
44. Sajid, M., Ansar, A., Hanif, A., Waheed, K., \& Tufail, S. (2017). Non Clinical Risk Factors of Myocardial Infarction: A Meta-Analysis Approach. J Biom Biostat, 8(4), 363.

45. Sajid, M. R., Muhammad, N., Zakaria, R., \& Shahbaz, A. (2019). Validation of Modified Dietary Habits Instrument in Cardiovascular Diseases Study. Paper presented at the Simposium Kebangsaan Sains Matematik ke-27 (SKSM 27), 26-27 November.

46. Scollo, M., \& Winstanley, M. (2016). Tobacco in Australia: facts and issues. Melbourne: Cancer Council Victoria; 2012.

47. Silva, A. A. M. d., Vasconcelos, A. G. G., Bettiol, H., \& Barbieri, M. A. (2010). Socioeconomic status, birth weight, maternal smoking during pregnancy and adiposity in early adult life: an analysis using structural equation modeling. Cadernos de Saude Publica, 26, 15-29.

48. Singh-Manoux, A., Marmot, M. G., \& Adler, N. E. (2005). Does subjective social status predict health and change in health status better than objective status? Psychosomatic Medicine, 67(6), 855861.

49. Song, H., Fang, F., Arnberg, F. K., Mataix-Cols, D., de la Cruz, L. F., Almqvist, C., . . . Valdimarsdóttir, U. A. (2019). Stress related disorders and risk of cardiovascular disease: population based, sibling controlled cohort study. BMJ, 365, 11255.

50. Statistics, P. B. o. (2017). Provisional Summary Results of 6th Population and Housing Census2017.

51. Thivel, D., Tremblay, A., Genin, P. M., Panahi, S., Rivière, D., \& Duclos, M. (2018). Physical Activity, Inactivity, and Sedentary Behaviors: Definitions and Implications in Occupational Health. Frontiers in public health, 6, 288.

52. Urbach, N., \& Ahlemann, F. (2010). Structural equation modeling in information systems research using partial least squares. Journal of Information technology theory and application, 11(2), 5-40.

53. Wareham, N. J., Jakes, R. W., Rennie, K. L., Schuit, J., Mitchell, J., Hennings, S., \& Day, N. E. (2003). Validity and repeatability of a simple index derived from the short physical activity questionnaire used in the European Prospective Investigation into Cancer and Nutrition (EPIC) study. Public health nutrition, 6(4), 407-413.

54. Wu, M.-C., Yang, Y.-C., Wu, J.-S., Wang, R.-H., Lu, F.-H., \& Chang, C.-J. (2012). Short sleep duration associated with a higher prevalence of metabolic syndrome in an apparently healthy population. Preventive Medicine, 55(4), 305-309.

55. Xi, B., Veeranki, S. P., Zhao, M., Ma, C., Yan, Y., \& Mi, J. (2017). Relationship of alcohol consumption to all-cause, cardiovascular, and cancer-related mortality in US adults. Journal of the American College of Cardiology, 70(8), 913-922. 